\title{
25 Research Square \\ Cullin3 Aggravates The Inflammatory Response of PDLSCs Via Regulation of Shh Signaling and Nrf2
}

\section{Wanhong Chen}

Quanzhou First Hospital

Jiangling Su

Quanzhou First Hospital

Shixiong Cai

Quanzhou First Hospital

Chun Shi ( $\boldsymbol{D}$ jskk_kkk231@163.com )

Dalian Medical College: Dalian Medical University https://orcid.org/0000-0003-3825-0948

\section{Research Article}

Keywords: P. gingivalis-LPS, Cullin3, Sonic Hedgehog, inflammation, PDLSCs

Posted Date: April 16th, 2021

DOI: https://doi.org/10.21203/rs.3.rs-368145/v1

License: (c) (1) This work is licensed under a Creative Commons Attribution 4.0 International License. Read Full License 


\section{Abstract}

Objective: Sonic Hedgehog (Shh) was found to be correlated with inflammation degree of patients with periodontitis. Cullin3 is an important ubiquitin ligase for controlling Shh signaling. In this study, we exerted ourselves to clarify the roles of Shh and Cullin3 in P. gingivalis-LPS (Pg-LPS)-treated periodontal ligament stem cells (PDLSCs).

Methods: Cell viability was detected using cell counting kit-8 (CCK-8). The inflammatory cytokines of PDLSCs were estimated by enzyme-linked immunosorbent assay (ELISA). The protein levels of Shh, Gli1 and NF-E2-related factor2 (Nrf2) were determined via western blots. Alkaline phosphatase staining and Alizarin red staining were performed to evaluate the differentiation and mineralization capabilities of PDLSCs. The apoptotic cells were screened by TUNEL staining.

Results: Pg-LPS inhibited cell viability and triggered inflammation of PDLSCs. Overexpression of Cullin3 impeded the differentiation and mineralization capabilities of PDLSCs. Moreover, Cullin3 overexpression aggravated inflammation and cell apoptosis induced by Pg-LPS. Of note, while the protein levels of Shh, Gli1 and Nrf2 were elevated in PDLSCs treated with Pg-LPS, overexpression of Cullin3 decreased the expressions of them.

Conclusion: Shh/Gli1 and Nrf2 were involved in the inflammation and cell apoptosis of PDLSCs, which was dominated by Cullin3.

\section{Introduction}

Periodontitis is a prevalent oral disease worldwide. Epidemiological survey shows that over $50 \%$ adults suffer from periodontal disease $(1,2)$. It is generally accepted that plaque bacteria, excessively accumulating on the tooth surface, invades periodontal tissue and triggers the inflammatory response, further resulting in the destruction of the periodontal tissue connection and the resorption of alveolar bone (3). Ultimately, the supporting structure of the tooth is lost (4). However, periodontal ligament has a good regeneration ability in physiological conditions in that alveolar bone and periodontal ligament are always in dynamic reconstruction, maintaining the integrity of periodontal support structure (5).

At present, there are rarely any effective therapies for periodontitis (6). The biological role of periodontal ligament stem cells (PDLSCs) is a hotspot in the treatment of periodontal disease. PDLSCs are crucial in maintaining the dynamic balance of periodontal tissues and are also the basis of periodontal tissue regeneration due to their multiple differentiation capabilities (7). Nevertheless, Osteogenic differentiation of PDLSCs was inhibited during the progression of periodontitis (8).

Sonic Hedgehog (Shh) is a member of Hedgehog $(\mathrm{Hh})$ family and is widely distributed in multiple organs (9). Hh signal is usually silent in adults, even though it is involved in tissue maintenance and regeneration by modulating the regeneration and differentiation of stem cells (10). High expression of Shh was 
observed in gingival crevicular fluid of patients with periodontitis (11), indicating that Shh may participate in the inflammatory response of periodontitis.

Cullin3 is a kind of E3 ubiquitin ligase with RING domain (12), which is different from other Cullins in E3 ligase family because it has no bridging protein. And a unique 3-box domain is needed to stabilize the binding sites. Therefore, there are not many active Cullin3 ubiquitin ligase complexes $(13,14)$. Cullin3 represented a common signaling node for controlling Shh signaling pathways (15). As a key transcription factor responsible for Shh signal, Gli1 could be degraded by Cullin3 $(16,17)$. In addition, Keleh-like ECHassociated protein 1 (Keap1) could interact with Cullin3, mediating the ubiquitination and degradation of NF-E2-related factor2 (Nrf2) $(18,19)$. Keap1/Nrf2 is a crucial signaling pathway that orchestrates inflammation and oxidative stress $(20,21)$. In this study, we aimed to illustrate the roles of Shh signaling pathways in LPS-induced PDLSCs and evaluate the effect of Cullin3 in the inflammatory process.

\section{Methods And Materials}

\section{Cell culture and transfection}

PDLSCs (LMAI Bio, Shanghai, China) were maintained in osteogenic-inducing medium containing 10\% FBS, $50 \mu \mathrm{M} / \mathrm{mL}$ ascorbic acid, $5 \mathrm{mM} \beta$-glycerophosphate and $100 \mathrm{nM}$ dexamethasone at $37^{\circ} \mathrm{C}$ in a $5 \%$ $\mathrm{CO}_{2}$ humidified incubator. $P$. gingivalis-LPS (Pg-LPS; Sigma-Aldrich, MA, USA) was used to stimulate PDLSCs as previously described $(22,23)$. Overexpression plasmids pcDNA 3.1-Cullin3 was generated by GenePharma (Shanghai, China). 1.2 $\mu \mathrm{g}$ pcDNA 3.1-Cullin3 was transfected into PDLSCs at a density of 2 $\times 10^{4}$ per well using Lipofectamine 3000 reagent (Invitrogen) according to the instruction.

\section{CCK-8 assay}

PDLSCs were seeded into a 96-well plate at a density of $1 \times 10^{3}$ per well. Cell viability was estimated at 0 $\mathrm{h}, 24 \mathrm{~h}$ and $48 \mathrm{~h}$ at first, after which $10 \mu \mathrm{l} \mathrm{CCK}-8$ solution (Dojindo, Kumamoto, Japan) was added into each well, followed by incubation for $1 \mathrm{~h}$. The absorbance value was at last recorded at $450 \mathrm{~nm}$.

\section{ELISA assay}

The supernatants of PDLSCs were collected and centrifuged at $4^{\circ} \mathrm{C}(1000 \mathrm{~g})$ for $10 \mathrm{~min}$. The concentration of TNF- $a, \mathrm{IL}-6, \mathrm{IL}-1 \beta$ and IL-10 was determined by ELISA kits (Beyotime, Jiangsu, China) according to the manufacturer's instruction. The absorbance value was recorded at $450 \mathrm{~nm}$.

\section{Western blots}

Total protein from cells were extracted using RIPA lysis buffer (Solarbio, Beijing, China). And the protein concentration was determined by a BCA assay kit (Beyotime, Jiangsu, China). Afterwards, SDS-PAGE was prepared to separate proteins, which were then transferred onto polyvinylidene difluoride (PVDF) membranes (EMD Millipore, MA, USA). PVDF membranes were incubated with primary antibodies, antiShh, anti-Gli1, anti-Cullin3 and anti-Nrf2 (Abcam, Cambridge, UK), which were removed the second day. 
And secondary antibodies (Sigma-Aldrich) were prepared for incubation with PVDF membranes. The protein bands were visualized and analyzed using a chemiluminescence system (Bio-Rad, CA, USA).

\section{ALP staining}

PDLSCs were seeded into a 24-well plate at a density of $2 \times 10^{4}$ per well. Osteogenic-inducing medium was prepared to culture PDLSCs for $7 \mathrm{~d}$. The medium was refreshed every 3 days, which was removed later on the 7th day. PDLSCS were subsequently cultured with added fix solution (MKBio, Shanghai, China) for $4 \mathrm{~min}$ at room temperature. Then with $0.6 \mathrm{ml}$ dye (MKBio) added into each well, the cells were cultured at room temperature for $10 \mathrm{~min}$ in the dark. Cell observation was done under a light microscope (Carl Zeiss, Jena, Germany) at last.

\section{Alizarin red staining}

The mineralization of PDLSCs was evaluated by Alizarin red S. PDLSCs were briefly seeded into a 24-well plate at a density of $2 \times 10^{4}$ per well. Osteogenic-inducing medium was prepared to culture PDLSCs for $21 \mathrm{~d}$, after which PDLSCs were fixed by $4 \%$ paraformaldehyde for $15 \mathrm{~min}$ at $4^{\circ} \mathrm{C}$. The cells were then incubated for 30 min with $0.2 \%$ Alizarin Red S solution (Sigma-Aldrich) added into each well. Lastly, cell observation was done under a light microscope (Carl Zeiss, Jena, Germany).

\section{TUNEL assay}

Apoptotic cells were detected by a TUNEL staining kit (KeyGEN, Jiangsu, China). 4\% paraformaldehyde was used to fix PDLSCs for $30 \mathrm{~min}$ at room temperature, after which Proteinase $\mathrm{K}$ was added for incubation for another $30 \mathrm{~min}$ at $37^{\circ} \mathrm{C}$. Subsequently, treatment of $1 \%$ Triton X-100 was added for incubation for $5 \mathrm{~min}$. The cells were then incubated likewise for $30 \mathrm{~min}$ at $37^{\circ} \mathrm{C}$ in the dark after Streptavidin-HRP was added to the plate. Color reaction was produced by adding DAB solution. And then the cells were re-dyed with hematoxylin. Finally, the cells were observed under a light microscope (Carl Zeiss, Jena, Germany).

\section{Statistical analysis}

Data was presented as mean \pm SD and was analyzed by GraphPad Prism 6.0. Student's t-test and oneway analysis of variance tests followed by Tukey's post hoc test were used to compare differences between groups. $\mathrm{P}<0.05$ was considered as statistical significance.

\section{Results}

\section{Pg-LPS impairs cell viability and triggers inflammtion of PDLSCs}

PDLSCs were challenged to different concentrations of Pg-LPS, the viability of which was examined after 24 and $72 \mathrm{~h}$. It was observed that cell viability slightly decreased when cells were exposed to $0.1 \mu \mathrm{g} / \mathrm{ml}$ Pg-LPS. Of note, cell viability was significantly reduced when the concentration of Pg-LPS reached 1 
$\mu \mathrm{g} / \mathrm{ml}$ (Fig. 1A). And Pg-LPS at $10 \mu \mathrm{g} / \mathrm{ml}$ represented more severe damage on cell viability. In addition, the productions of pro-inflammatory cytokines including TNF-a, IL- 6 and IL-1 $\beta$ were elevated when PDLSCs were exposed to $1 \mu \mathrm{g} / \mathrm{ml}$, whereas the level of anti-inflammatory cytokines IL-10 also increased following exposure to $0.1 \mu \mathrm{g} / \mathrm{ml}$ or $1 \mu \mathrm{g} / \mathrm{ml} \mathrm{Pg-LPS} \mathrm{(Fig.} \mathrm{1B).} \mathrm{However,} \mathrm{it} \mathrm{was} \mathrm{noticed} \mathrm{that} \mathrm{IL-10} \mathrm{was}$ markedly reduced when the concentration of Pg-LPS reached $10 \mu \mathrm{g} / \mathrm{ml}$, indicating that high concentration of Pg-LPS triggered predominantly the secretions of pro-inflammatory cytokines.

\section{Shh signaling and Nrf2 are activated in Pg-LPS-treated PDLSCs}

Furthermore, it was found that the protein levels of Shh and Gli1 were elevated when cells were exposed to Pg-LPS, accompanied by the increase of Nrf2 (Fig. 2A and 2B), suggesting a stressful increase of Shh, Gli1 and Nrf2 in response to Pg-LPS challenge. Given that Cullin3 plays a vital role in degrading Gli1 and Nrf2, overexpression plasmids pcDNA 3.1-Cullin3 was transfected into PDLSCs. Results showed that pcDNA 3.1-Cullin3 effectively upregulated the expression of Cullin3 (Fig. 3A). Overexpression of Cullin3 further impaired cell viability in the presence of $1 \mu \mathrm{g} / \mathrm{ml} \mathrm{Pg-LPS} \mathrm{(Fig.} \mathrm{3B).}$

\section{Differentiation and mineralization of PDLSCs are inhibited by Cullin3 overexpression}

The differentiation and mineralization capabilities of PDLSCs were estimated. The differentiation capability of PDLSCs induced by osteogenic-inducing medium was weakened in response to $1 \mu \mathrm{g} / \mathrm{ml} \mathrm{Pg}$ LPS. And overexpression of Cullin3 further impaired the differentiation capability (Figure. 4A). Consistent result was observed via assessing the mineralization capability of PDLSCs (Figure. 4B), that Cullin3 overexpression could impede PDLSCs mineralization.

\section{Overexpression of Cullin3 exacerbates inflammation and apoptosis}

The inflammatory response of PDLSCs following transfection of pcDNA 3.1-Cullin3 was evaluated using ELISA kits. It was noticed that $1 \mu \mathrm{g} / \mathrm{ml}$ Pg-LPS triggered high levels of TNF-a, IL- 6 and IL-1 $\beta$, and Cullin3 exacerbated the inflammatory response. By contrast, the level of IL-10 significantly decreased following transfection of pcDNA 3.1-Cullin3 (Fig. 5A). Moreover, TUNEL staining demonstrated that LPS induced cell apoptosis was further promoted by Cullin3 overexpression (Fig. 5B and 5C). Additionally, it was found that Shh, Gli1 and Nrf2 were upregulated in response to $1 \mu \mathrm{g} / \mathrm{ml} \mathrm{Pg-LPS}$, while Cullin3 overexpression ameliorated these effects (Fig. 6A and 6B), indicating that Cullin3 promoted inflammatory response and cell apoptosis likely through downregulating the expression of Shh, Gli1 and Nrf2.

\section{Discussion}


PDLSCs have the ability of self-renewal and differentiation, which play a major role in repairing damaged dental tissues and are considered to be most useful for periodontal reconstruction and regeneration (24). In the current study, we found that cell viability of PDLSCs was significantly reduced when exposed to 1 $\mu \mathrm{g} / \mathrm{ml} \mathrm{Pg-LPS}$. Furthermore, numerous pro-inflammatory cytokines including TNF-a, IL-6 and IL-1 $\beta$ were produced following stimulation of Pg-LPS. Shh is reported to be potentially a new therapeutic target for periodontitis and periodontal regeneration (25), the expression of which along with that of Gli1 in PDLSCs was examined in this study and was found to have an obvious increase after Pg-LPS stimulation.

In basal condition, Keap1 binds Nrf2 to its BTB domain, and connects Cullin3 to its Kelch domain. Nrf2 is maintained at a low level through ubiquitination degradation mediated by Cullin3 (18). NRF escapes from degradation in response to stimulus and is thus released, regulating the expression of downstream target genes (26). Our results showed that the expression of Nrf2 increased in response to Pg-LPS stimulation. It was also noticed that overexpression of Cullin3 in PDLSCs impaired cell viability and inhibited the differentiation and mineralization of PDLSCs. Moreover, inflammatory response was exacerbated and apoptotic cells increased following Cullin3 overexpression in the presence of Pg-LPS. Of note, the levels of Shh, Gli1 and Nrf2 in PDLSCs decreased when transfected with pcDNA3.1-Cullin3, suggesting that Shh/Gli1 and Nrf2 mediated by Cullin3 might participate in the inflammatory response, and thus affect the differentiation and mineralization of PDLSCs.

In summary, Cullin3 was found to be involved in the inflammatory response of PDLSCs via orchestrating the expression of Shh signaling and Nrf2. However, the present study is limited by inadequate exploration of the nuclear regulation of Nrf2. And it is of necessity to further investigate the specific regulatory mechanism of Gli1 in future study.

\section{Declarations}

\section{Ethics approval and consent to participate}

Not Applicable.

\section{Consent for publication}

All authors agreed to publish.

\section{Availability of data and materials}

The data that support the findings of this study are available from the corresponding author upon reasonable request.

\section{Competing interests}

The authors declare that they have no competing interests.

\section{Funding}


This work was supported by grants from the Liaoning Natural Science Foundation (No.20180550563 to Correspondence).

\section{Authors' contributions}

Chun Shi contributed to conception and design, analysis and interpretation of the data, critically revised the article for important intellectual content. Wanhong Chen contributed to design and analysis of the data, drafted and revised the manuscript. Jiangling Su and Shixiong Cai substantially contributed to conception and design, acquisition, analysis, and interpretation of data; drafted and critically revised the article for important intellectual content. All authors approved the final version of the article and agreed to be accountable for all aspects of the work in ensuring that questions related to the accuracy or integrity of any part of the work are appropriately investigated and resolved.

\section{Acknowledgements}

Not applicable.

\section{References}

1. Liu, J., J. Ruan, M.D. Weir et al Periodontal Bone-Ligament-Cementum Regeneration via Scaffolds and Stem Cells. Cells 2019; 8(6).

2. Carasol, M., J.C. Llodra, A. Fernández-Meseguer et al Periodontal conditions among employed adults in Spain. J Clin Periodontol 2016; 43(7): 548-56.

3. Van Dyke, T.E. The management of inflammation in periodontal disease. J Periodontol 2008; 79(8 Suppl): $1601-8$.

4. Chen, F.M., H.H. Sun, H. Lu,Q. Yu Stem cell-delivery therapeutics for periodontal tissue regeneration. Biomaterials 2012; 33(27): 6320-44.

5. Fukushima, H., H. Kajiya, K. Takada, F. Okamoto,K. Okabe Expression and role of RANKL in periodontal ligament cells during physiological root-resorption in human deciduous teeth. Eur $\mathrm{J}$ Oral Sci 2003; 111(4): 346-52.

6. Cionca, N. Editorial: Use and Misuse of Systemic Antibiotics in Periodontitis Treatment. Oral Health Prev Dent 2017; 15(4): 305-306.

7. Seo, B.M., M. Miura, S. Gronthos et al Investigation of multipotent postnatal stem cells from human periodontal ligament. Lancet 2004; 364(9429): 149-55.

8. Park, J.C., J.M. Kim, I.H. Jung et al Isolation and characterization of human periodontal ligament (PDL) stem cells (PDLSCs) from the inflamed PDL tissue: in vitro and in vivo evaluations. J Clin Periodontol 2011; 38(8): 721-31.

9. Bitgood, M.J.,A.P. McMahon Hedgehog and Bmp genes are coexpressed at many diverse sites of cellcell interaction in the mouse embryo. Dev Biol 1995; 172(1): 126-38.

10. Petrova, R.,A.L. Joyner Roles for Hedgehog signaling in adult organ homeostasis and repair. Development 2014; 141(18): 3445-57. 
11. Zhi-fen, C., C. Lian-bao,X. Yan-hua Expression of IL-6 and Shh in Gingival Crevicular Fluid of Patients with Peri-implantitis. journal of oral science research 2018.

12. Sarikas, A., T. Hartmann,Z.Q. Pan The cullin protein family. Genome Biol 2011; 12(4): 220.

13. Furukawa, M., Y.J. He, C. Borchers,Y. Xiong Targeting of protein ubiquitination by BTB-Cullin 3-Roc1 ubiquitin ligases. Nat Cell Biol 2003; 5(11): 1001-7.

14. Smaldone, G., L. Pirone, N. Balasco et al Cullin 3 Recognition Is Not a Universal Property among KCTD Proteins. PLoS One 2015; 10(5): e0126808.

15. Jin, X., H.M. Jeon, X. Jin et al The ID1-CULLIN3 Axis Regulates Intracellular SHH and WNT Signaling in Glioblastoma Stem Cells. Cell Rep 2016; 16(6): 1629-1641.

16. Heride, C., D.J. Rigden, E. Bertsoulaki et al The centrosomal deubiquitylase USP21 regulates Gli1 transcriptional activity and stability. J Cell Sci 2016; 129(21): 4001-4013.

17. Ou, C.Y., C.H. Wang, J. Jiang,C.T. Chien Suppression of Hedgehog signaling by Cul3 ligases in proliferation control of retinal precursors. Dev Biol 2007; 308(1): 106-19.

18. Iso, T., T. Suzuki, L. Baird,M. Yamamoto Absolute Amounts and Status of the Nrf2-Keap1-Cul3 Complex within Cells. Mol Cell Biol 2016; 36(24): 3100-3112.

19. Tao, S., P. Liu, G. Luo et al p97 Negatively Regulates NRF2 by Extracting Ubiquitylated NRF2 from the KEAP1-CUL3 E3 Complex. Mol Cell Biol 2017; 37(8).

20. Huang, J.L., C. Yu, M. Su et al Probucol, a "non-statin" cholesterol-lowering drug, ameliorates Dgalactose induced cognitive deficits by alleviating oxidative stress via Keap1/Nrf2 signaling pathway in mice. Aging (Albany NY) 2019; 11(19): 8542-8555.

21. Zhao, X.J., H.W. Yu, Y.Z. Yang et al Polydatin prevents fructose-induced liver inflammation and lipid deposition through increasing miR-200a to regulate Keap1/Nrf2 pathway. Redox Biol 2018; 18: 124137.

22. Du, A., S. Zhao, L. Wan et al MicroRNA expression profile of human periodontal ligament cells under the influence of Porphyromonas gingivalis LPS. J Cell Mol Med 2016; 20(7): 1329-38.

23. Yan, B., H. Zhang, T. Dai et al Necrostatin-1 promotes ectopic periodontal tissue like structure regeneration in LPS-treated PDLSCs. PLoS One 2018; 13(11): e0207760.

24. Hu, L., Y. Liu,S. Wang Stem cell-based tooth and periodontal regeneration. Oral Dis 2018; 24(5): 696705.

25. Bae, W.J., Q.S. Auh, H.C. Lim et al Sonic Hedgehog Promotes Cementoblastic Differentiation via Activating the BMP Pathways. Calcif Tissue Int 2016; 99(4): 396-407.

26. Su, C., P. Zhang, X. Song et al Tetrachlorobenzoquinone activates Nrf2 signaling by Keap1 crosslinking and ubiquitin translocation but not Keap1-Cullin3 complex dissociation. Chem Res Toxicol 2015; 28(4): 765-74.

\section{Figures}




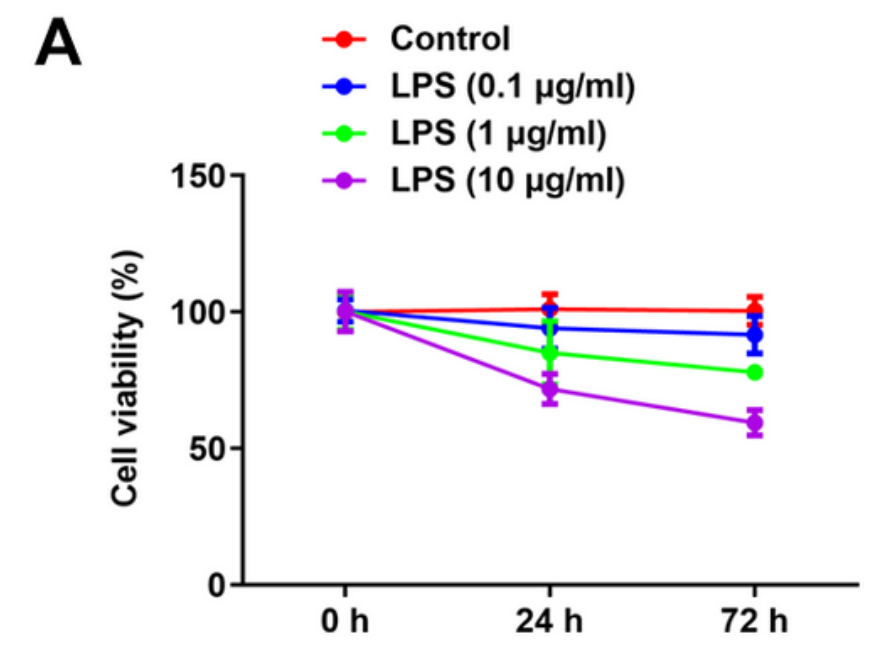

B
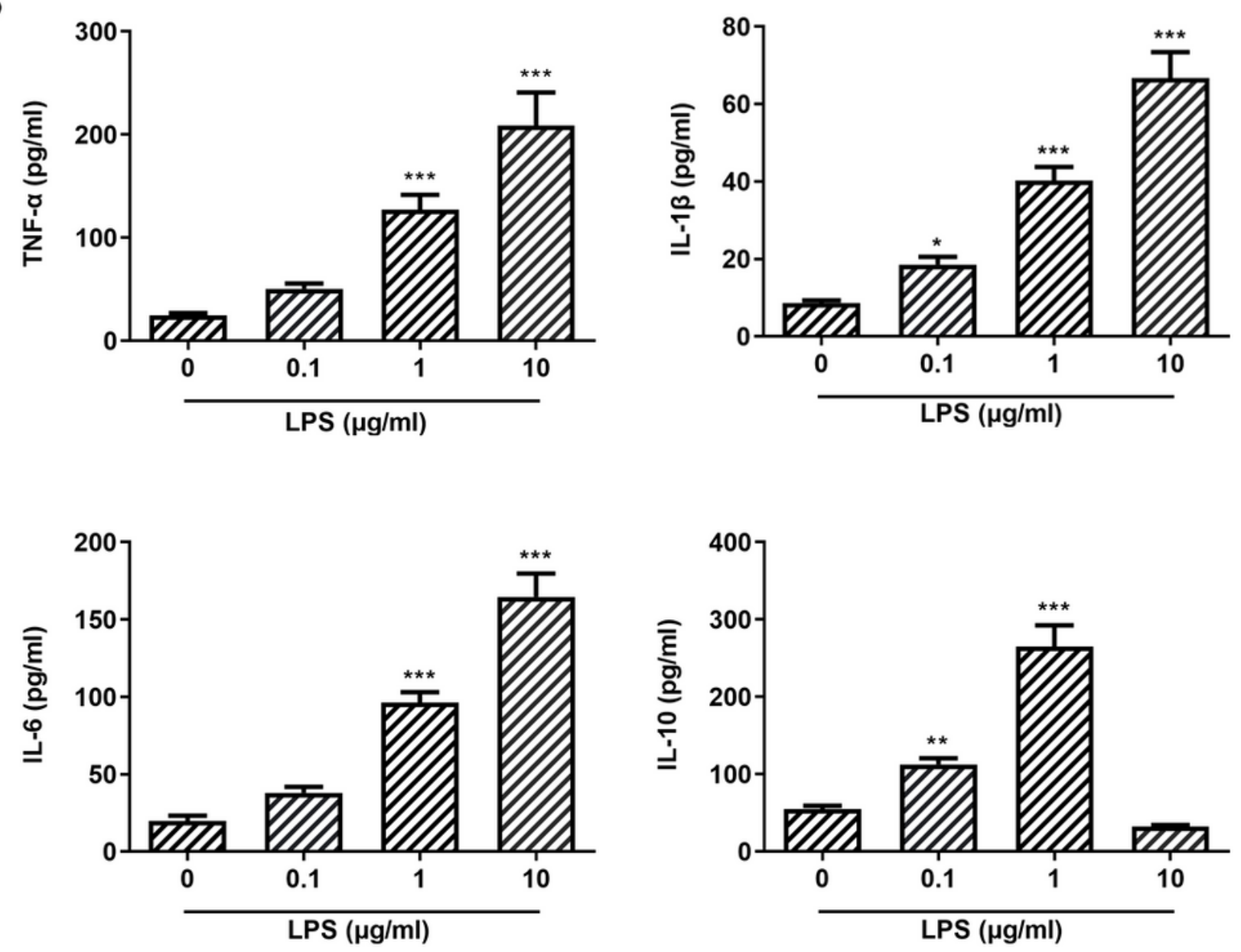

Figure 1

Pg-LPS impairs cell viability and triggers inflammtion of PDLSCs. (A) Effects of different concentrations of pg-LPS on the activity of PDLSCs were detected by CCK-8 assay. (B) Effects of different concentrations of pg-LPS on inflammatory factors (TNF- $\alpha$, IL-6, IL-1 $\beta$ and IL-10) in PDLSCs were detected by ELISA assay. ${ }^{\star} \mathrm{p}<0.05,{ }^{\star \star} \mathrm{p}<0.01$ and ${ }^{* \star *} \mathrm{p}<0.001 \mathrm{vs.} 0 \mu \mathrm{g} / \mathrm{ml}$. 
A
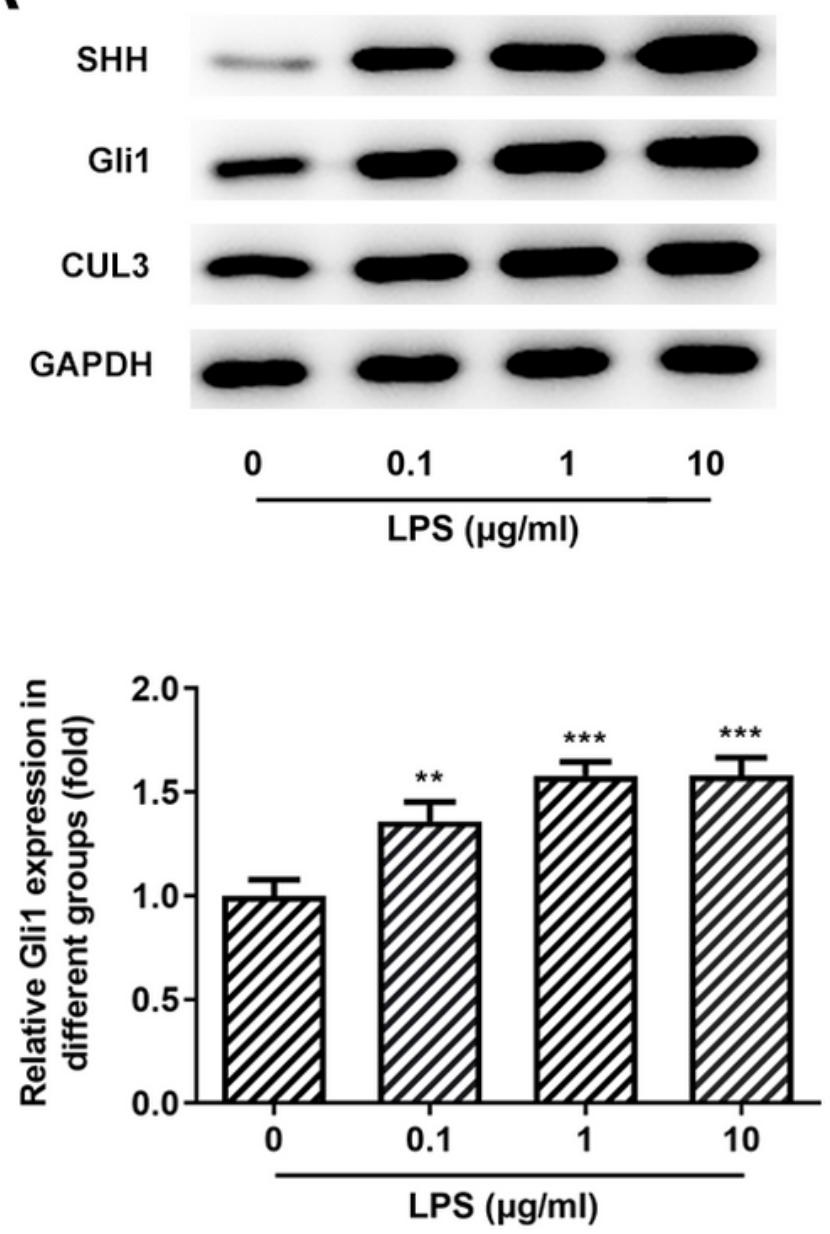

B

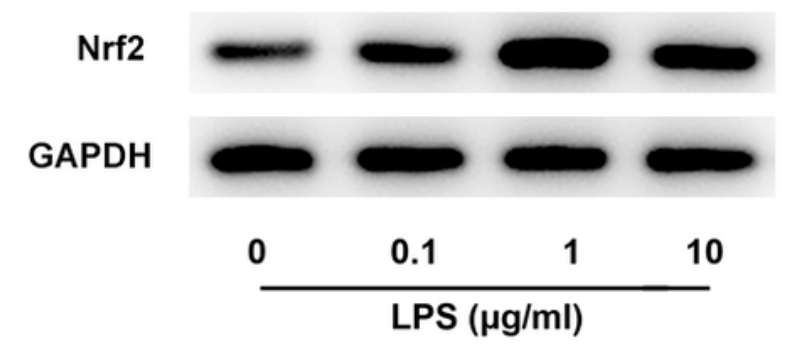

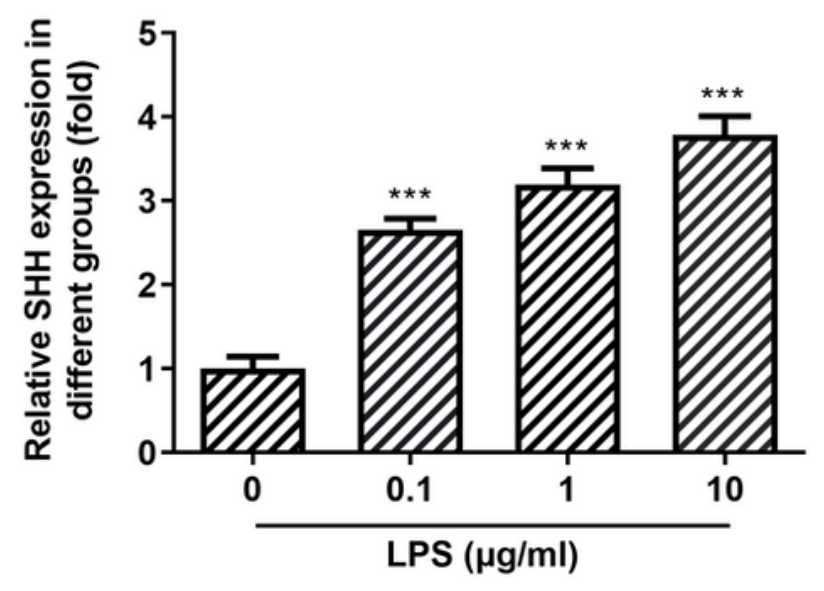
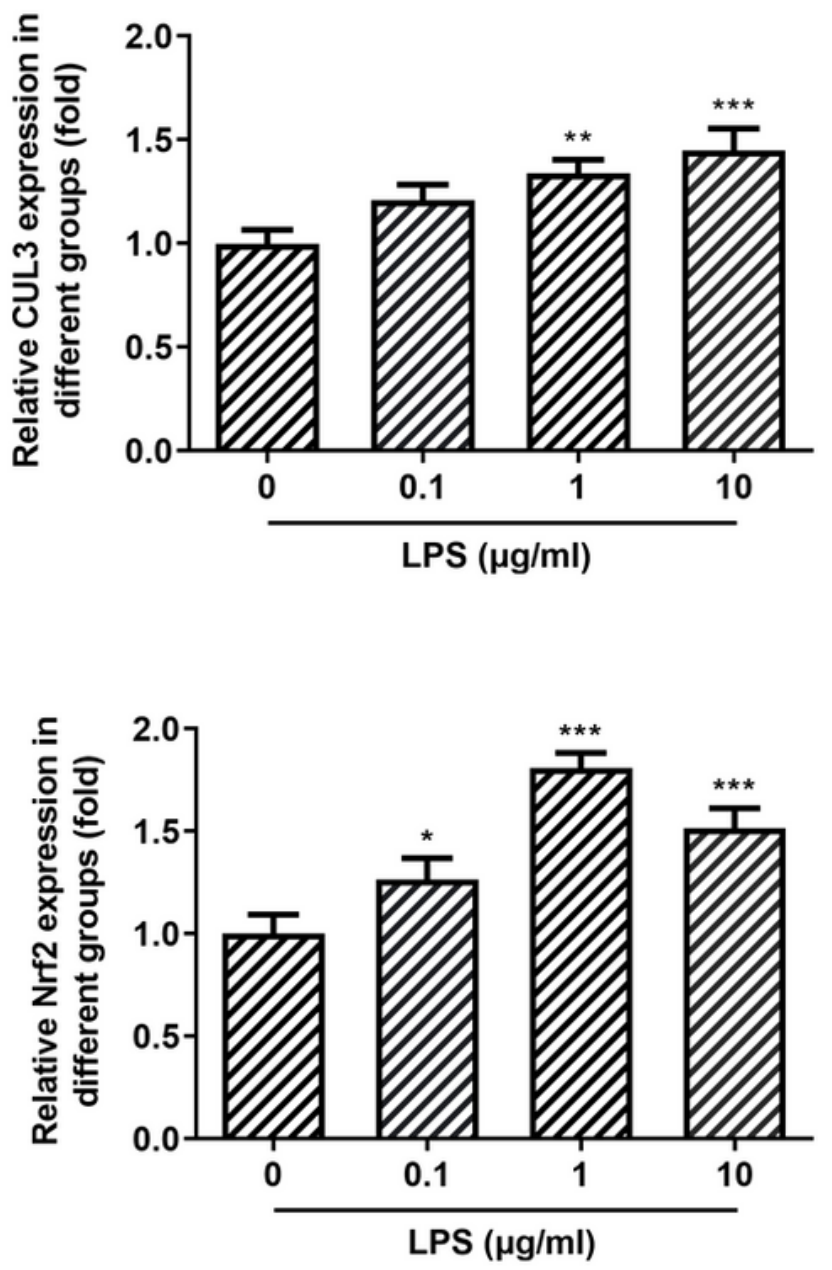

Figure 2

Effects of pg-LPS on SHH pathway-related proteins and Nrf2 in PDLSCs. (A) Western blot analysis was used to detect the effect of pg-LPS on SHH, Gli1 and Cullin3 in PDLSCs. (B) Western blot analysis was used to detect the effect of pg-LPS on Nrf2 in PDLSCs. ${ }^{*} p<0.05,{ }^{*} p<0.01$ and ${ }^{* *} p<0.001 \mathrm{vs} .0 \mu \mathrm{g} / \mathrm{ml}$. 


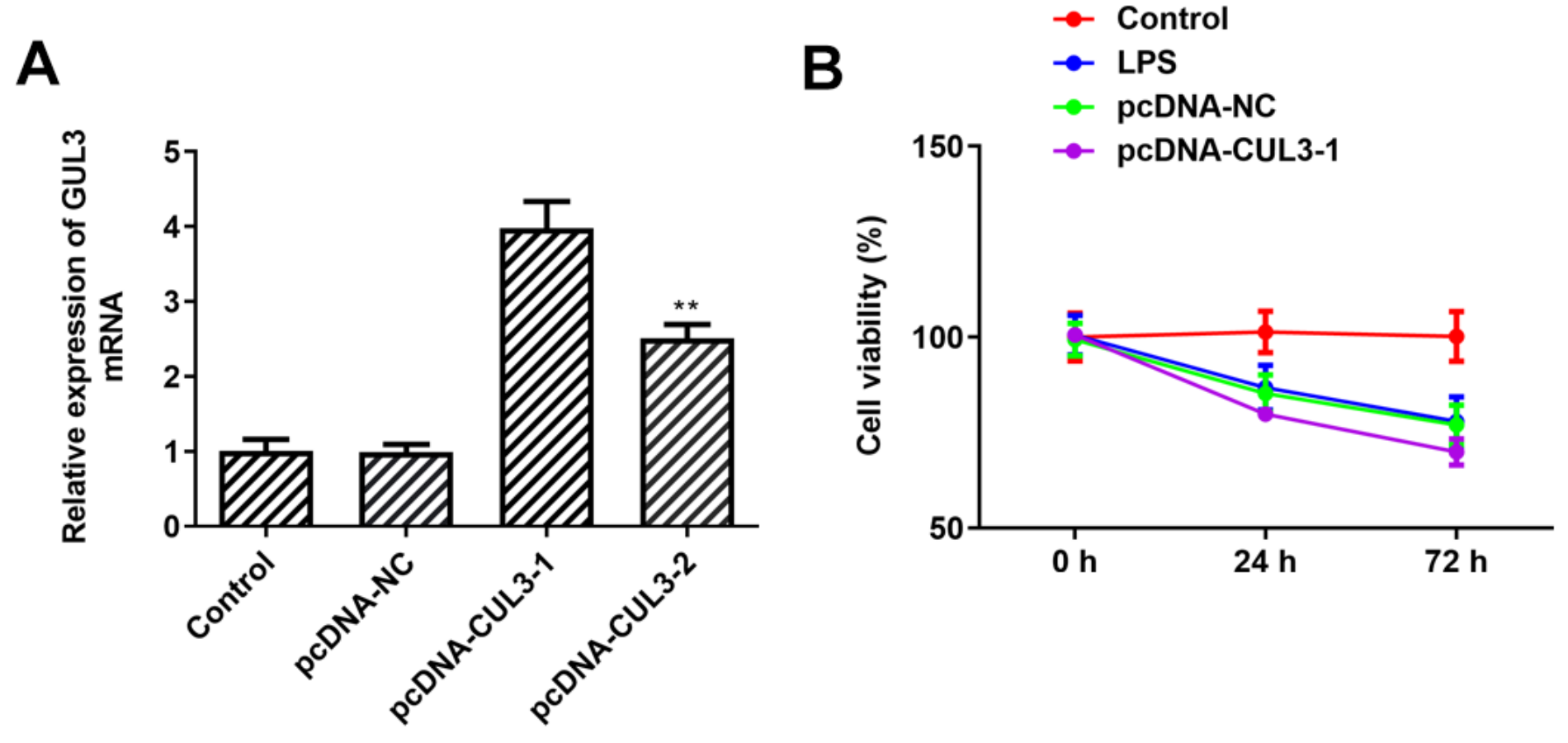

Figure 3

Cullin3 overexpression inhibits the activity of PDLSCs treated with pg-LPS. (A) The Cullin3 overexpression efficiency was detected by qRT-PCR after transfection. (B) Effects of Cullin3 overexpression on the activity of PDLSCs treated with pg-LPS were detected by CCK-8 assay. ${ }^{* *} \mathrm{p}<0.01$ vs. pcDNA-CUL3-1.

B

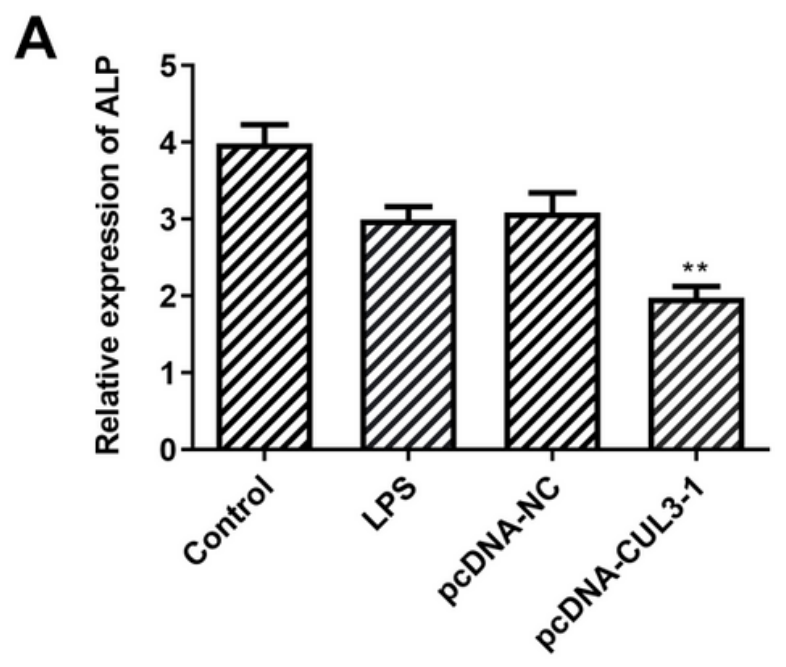

Control

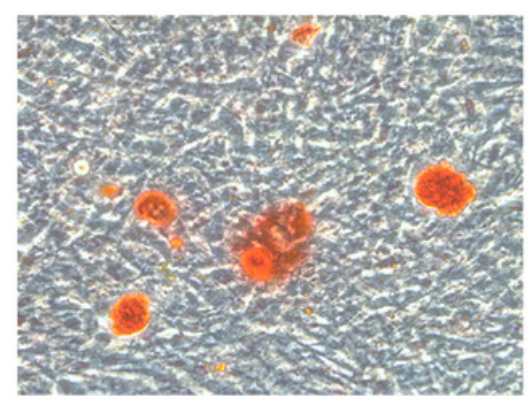

pcDNA-NC

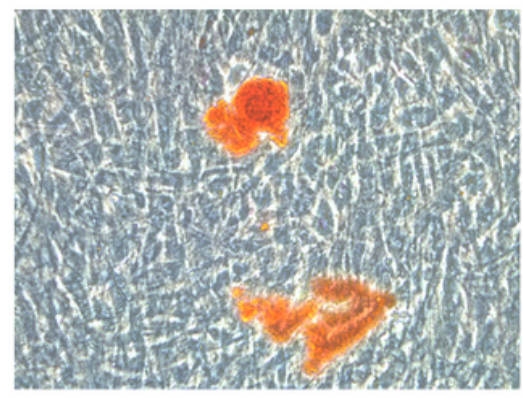

LPS

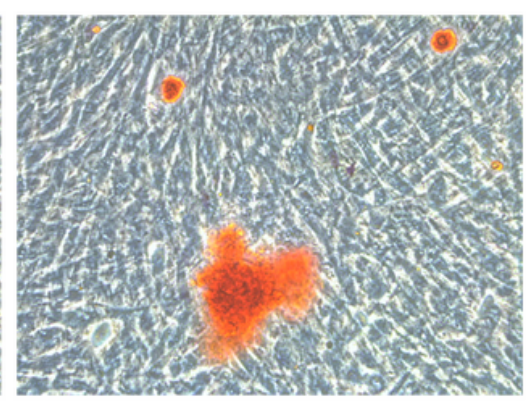

pCDNA-CUL3-1

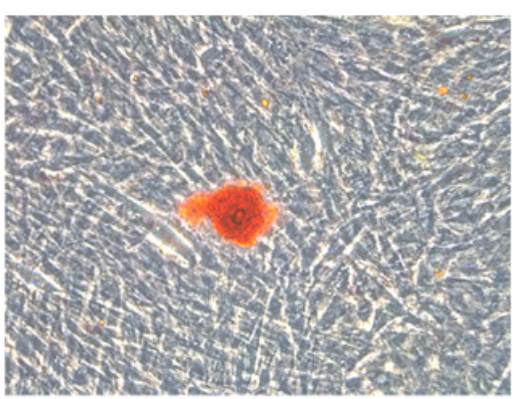

Figure 4 
Differentiation and mineralization of PDLSCs are inhibited by Cullin3 overexpression. (A) Effect of Cullin3 overexpression on cell differentiation was detected by ALP. (B) Effect of Cullin3 overexpression on cell mineralization was detected by alizarin red staining. ${ }^{*} p<0.01$ vs. pcDNA-NC.

A
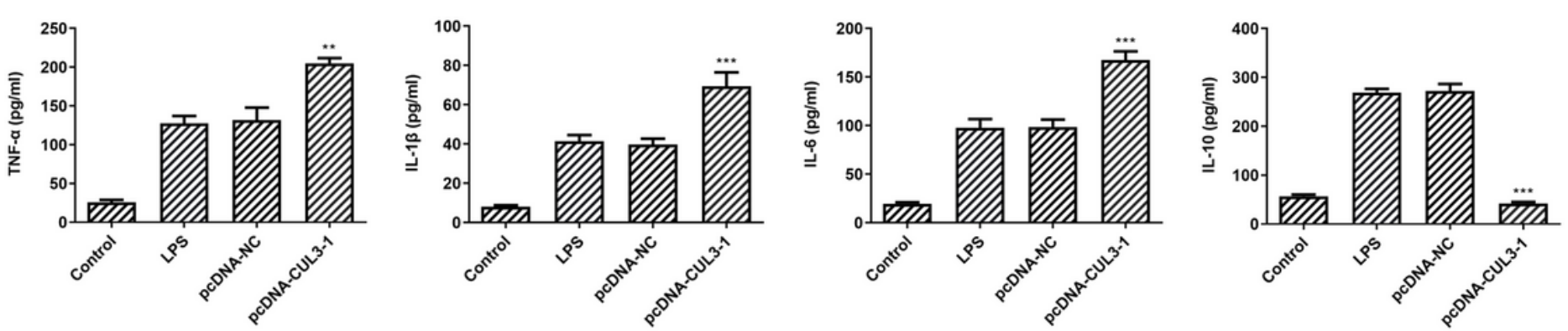

B

Control

pcDNA-NC

pcDNA-CUL3-1

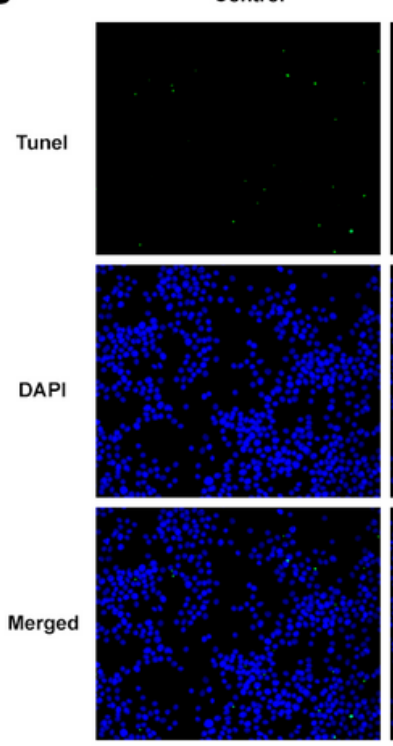

LPS
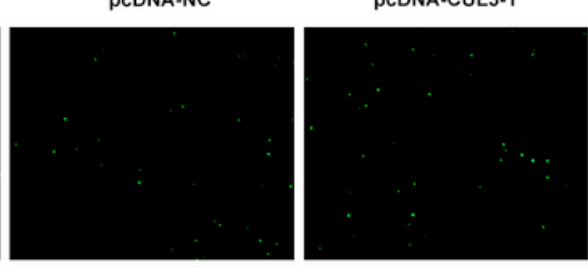

C
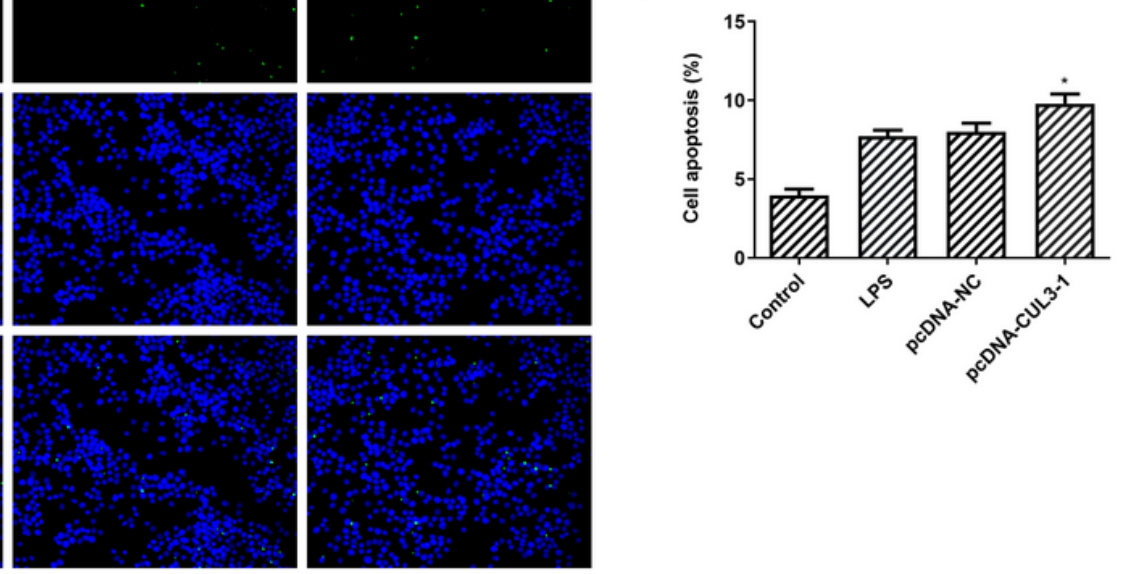

Figure 5

Cullin3 overexpression promotes inflammation and apoptosis of PDLSCstreated with pg-LPS. (A) ELISA assay was performed to detect the effect of Cullin3 overexpression on inflammatory factors (TNF- $a$, IL-6, IL-1 $\beta$ and IL-10) in PDLSCs treated with pg-LPS. (B) TUNEL and DAPI staining were performed to detect the effect of Cullin3 overexpression on apoptosis of PDLSCs treated with pg-LPS. (C) The percentage of apoptotic cells in each group was measured by TUNEL assay. ${ }^{\star} p<0.05,{ }^{*} p<0.01$ and ${ }^{* \star *} p<0.001$ vs. pcDNA-NC. 
A
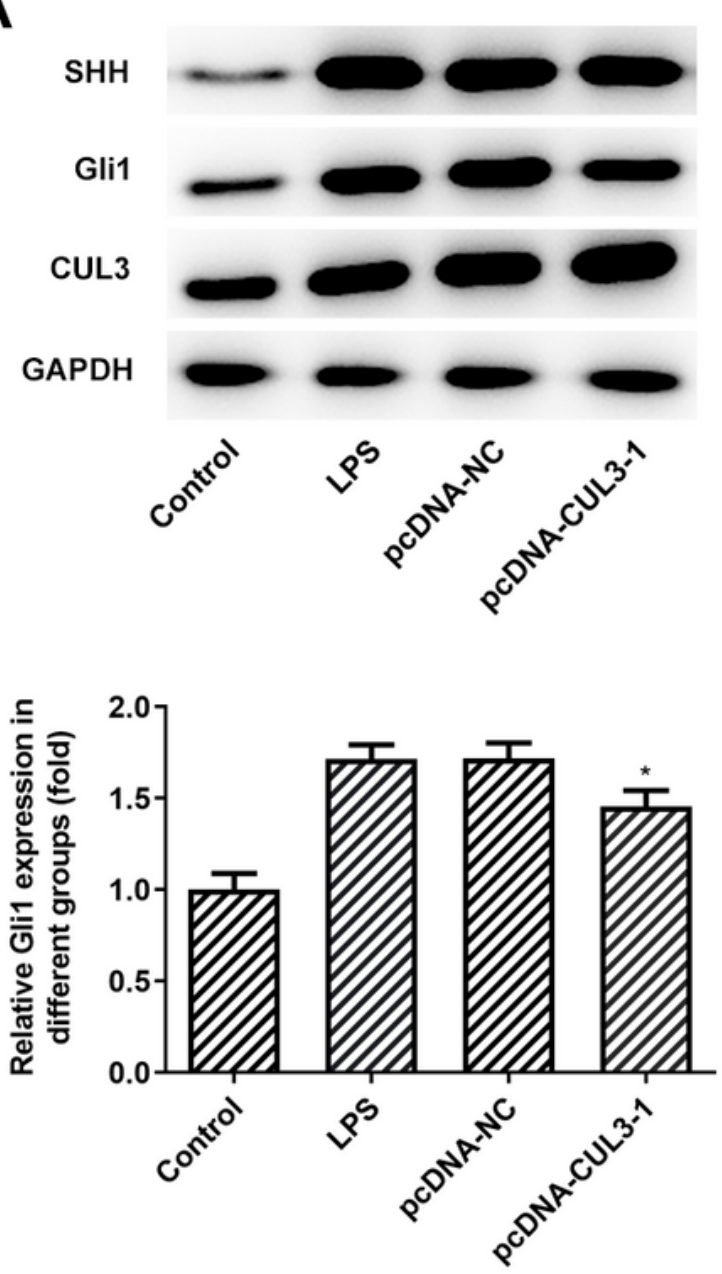

B

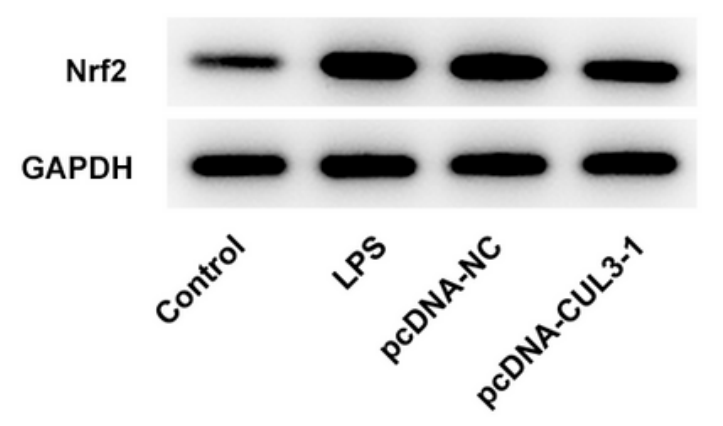

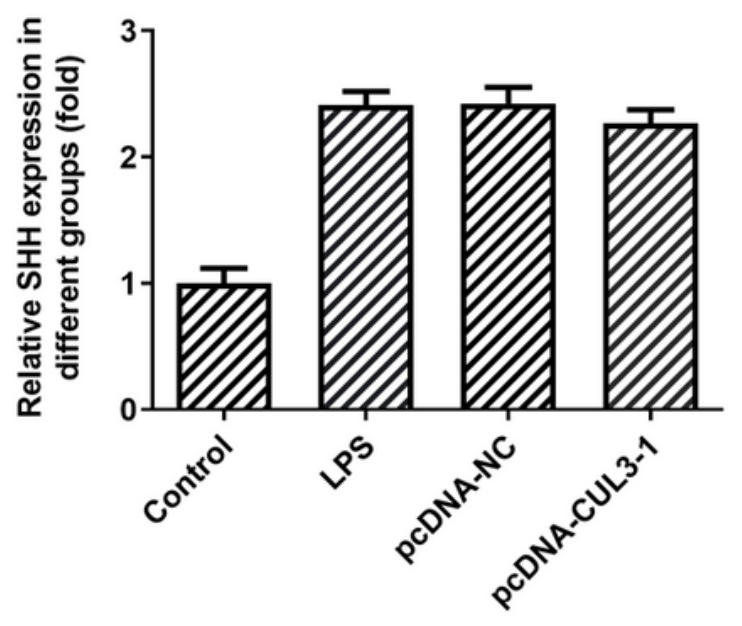
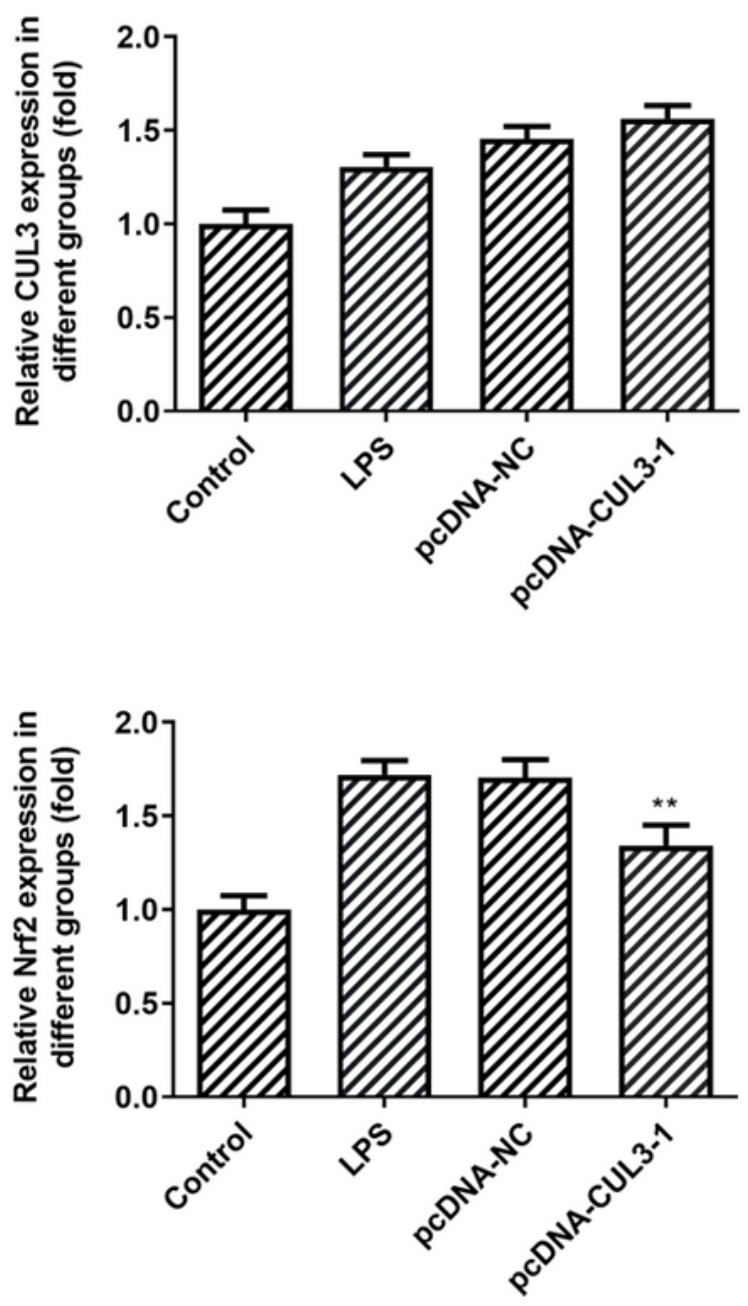

Figure 6

Effects of Cullin3 overexpression on SHH pathway-related proteins and Nrf2 in PDLSCs treated with pgLPS. (A) Western blot analysis was used to detect the effect of Cullin3 overexpression on SHH, Gli1 and Cullin3 in PDLSCs treated with pg-LPS. (B) Western blot analysis was used to detect the effect of Cullin3 overexpression on Nrf2 in PDLSCs treated with pg-LPS. ${ }^{*} p<0.05$ and ${ }^{* \star} p<0.01$ vs. pcDNA-NC. 